\title{
Interfacial hoop stress and instability of viscoelastic free surface flows
}

\author{
Michael D. Graham ${ }^{\mathrm{a})}$ \\ Department of Chemical Engineering, University of Wisconsin-Madison, 1415 Engineering Drive, Madison, \\ Wisconsin 53706-1691
}

(Received 29 July 2002; accepted 24 February 2003; published 6 May 2003)

\begin{abstract}
Viscoelasticity can dramatically exacerbate free surface flow instabilities. It is shown here that this destabilization arises, at least in part, from the combination of large tangential tension and curvature along a concave free surface. Tension and curvature generically lead to an unstable stress gradient at the free surface; a perturbation that locally thickens a fluid layer leads to a local accumulation of hoop stress, which drives further thickening of the layer. A simple theory based in this observation captures several features of experimental observations of coating flows, specifically the correlation between destabilization and extensional viscosity and the increase in wavenumber with viscoelasticity. In a particular model situation, instability of flow with a concave free surface can be reduced to a viscoelastic Rayleigh-Taylor problem, which is amenable to analytical treatment. In this situation the growth rate can be very large, because the stored elastic energy cannot balance the surface work associated with interface deformation. Application of the theory to the instability of filament stretching flow yields a prediction of the Weissenberg number below which instability does not occur that agrees well with experimental observations. (C) 2003 American Institute of Physics. [DOI: $10.1063 / 1.1568340]$
\end{abstract}

\section{INTRODUCTION}

Viscous free surface flows, such as those used in coating processes, are susceptible to interfacial instabilities. Although these instabilities are present in Newtonian fluids, they are often dramatically exacerbated by viscoelasticity. Figure 1 shows schematics of a number of such flows; they all have a concave free surface and their instabilities are characterized by the formation of "ribs" or "fibrils"interface structures that are extended in the mean flow direction and periodic in the transverse, or spanwise direction. In the ribbing instability of coating flows, the ribs appear above a critical value of the capillary number $C a=\eta U / \gamma$, where $\eta$ and $\gamma$ are viscosity and surface tension and $U$ a characteristic fluid velocity. In models of roll coating flows, this critical value $C a_{c}$ decreases monotonically and significantly when the fluid is viscoelastic. ${ }^{1-7}$ In slot coating, the limited evidence available suggests that flow of sufficiently dilute polyacrylamide solutions is actually more stable than flow of the solvent alone, perhaps due to shear-thinning, but that flow of more concentrated solutions, which have appreciable elasticity, is significantly destabilized. ${ }^{8}$ Destabilization was not observed for CMC solutions, which had negligible elasticity. Finally, a dramatic example of a fibril-forming instability in an elastic liquid occurs at high strains at the endplates of a filament stretching rheometer. ${ }^{9}$ In a filament stretching device, fluid is placed in the gap between two coaxial circular disks. ${ }^{10,11}$ The disks are then rapidly separated, and the fluid forms a nominally axisymmetric bridge that is very nearly cylindrical, except near the ends, where the flow is con-

${ }^{a)}$ Electronic mail: graham@engr.wisc.edu strained by the no-slip boundary conditions on the disks. Under some conditions, this bridge loses axisymmetry quite dramatically, as illustrated by Spiegelberg and McKinley ${ }^{9}$ - near the endplates, the filament splits into multiple fibrils and at later times the individual fibrils undergo a further splitting. Similar "elastic fingering" or "fibrillation" instabilities are observed when separating solid surfaces that are bridged by a soft elastic solid such as a pressure sensitive adhesive (see, e.g., Ref. 12).

To develop a foundation for understanding the instabilities in all of these cases, we first appeal to the canonical viscous free surface instability, the viscous fingering, or Saffman-Taylor instability. ${ }^{13}$ This arises when a viscous liquid is displaced from the gap between parallel plates-a Hele-Shaw cell — by a less viscous fluid such as air. The steady displacement by air of a Newtonian liquid from a Hele-Shaw cell is unstable because a small perturbation of the interface toward the liquid phase leads to a decreased viscous resistance to flow there. ${ }^{14}$ The pressure in the air is uniform, so the decreased flow resistance in the fluid leads to a higher velocity at the perturbation, causing its amplitude to increase. The first analysis of this phenomenon was given by Saffman and Taylor. ${ }^{13}$ The key term in the interfacial normal stress balance is the steady state stress gradient at the interface; Saffman and Taylor estimated this as the bulk pressure gradient, which is easily calculated. Park and Homsy ${ }^{15}$ refined the Saffman-Taylor analysis using matched asymptotic expansions, to obtain a rigorous expression for the depthaveraged pressure gradient at the interface. Employing this result in a linear stability analysis of the depth-averaged (i.e., Darcy's law) problem leads to good agreement between theory and experiments for Newtonian liquids. ${ }^{16}$ Pearson ${ }^{17}$ 


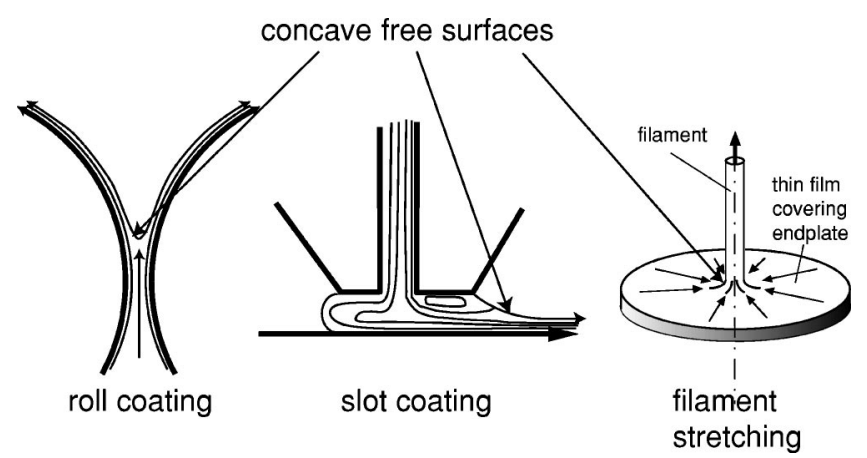

FIG. 1. Schematics of several archetypal free surface flows. Nominally, the roll coating and slot coating flows are two-dimensional, while the filament stretching flow is axisymmetric.

exploited the similarity between the flow in a Newtonian forward roll-coating operation and that in a tapered HeleShaw cell to elucidate the basic mechanism and provide a linear stability theory for the ribbing instability of Newtonian coating flows. Finally, full scale computational approaches for Newtonian flow have progressed to the point that in Newtonian forward roll coating, not only can the linear instability threshold be predicted (see, e.g., Refs. 18-21), but the fully nonlinear three-dimensional structure of ribs can also be captured. ${ }^{22}$

To attempt to understand how free surface instabilities are affected by viscoelasticity, several authors have built heuristic arguments that begin with the Saffman-Taylor mechanism. Specifically, Spiegelberg and McKinley ${ }^{9}$ suggested that a generalization of this mechanism might be responsible for the filament stretching instability. Many studies of peeling instabilities (i.e., fibril formation) in elastic adhesives refer either to the original Saffman-Taylor mechanism or to a paper of McEwan and Taylor, ${ }^{23}$ which treats the adhesive as a purely viscous liquid and modifies the Saffman-Taylor analysis to a peeling geometry. There are several stumbling blocks, however, to constructing a theory of free surface instability in viscoelastic flows. Simply evaluating the stress gradient at the interface based on a bulk estimate ${ }^{24}$ ignores dramatic changes in stress, and stress gradients, that can arise near a free surface-we elaborate on these effects below. Rigorous asymptotic approaches such as that of Ro and Homsy, ${ }^{25}$ although illuminating, are limited to small degrees of elasticity. Finally, computations of strongly viscoelastic flows are challenging - few computational stability analyses have been performed for highly elastic free surface flow, ${ }^{26-28}$ and only recently have steady two-dimensional solutions been presented for coating flows with a significant degree of elasticity. ${ }^{29,30}$ Therefore, in the present work we rely on general kinematic and dynamic properties of flows near free surfaces to extract key features of the mechanism of viscoelastic free surface flow instabilities.

This paper illustrates one direct way that viscoelasticity enters into the stability problem for a free surface flow, showing specifically how viscoelasticity modifies the unstable pressure gradient argument of the Saffman-Taylor mechanism via hoop stresses at a concave interface. This analysis yields a dimensionless group that characterizes the

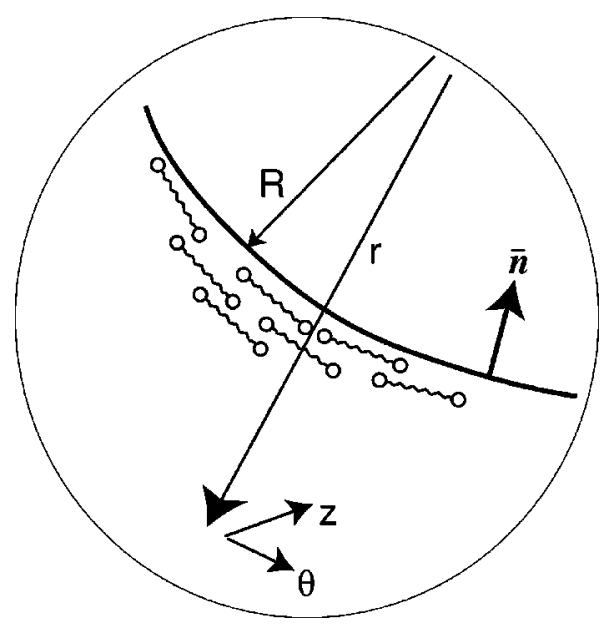

FIG. 2. Blowup of flow near a concave free surface like those in the flows of Fig. 1. The elongated dumbbells represent polymer molecules that are strongly stretched in the extensional flow near the interface.

susceptibility of a viscoelastic free surface flow to instability. In one idealized case, a nearly flat "transition region" in a coating flow, we can use a modified Rayleigh-Taylor analysis to further elucidate the effect of viscoelasticity on free surface instabilities. Finally, we revisit the filament stretching instability, finding that it is possible to capture some features of the phenomenon within the present theory.

\section{STRESS AT A CURVED FREE SURFACE}

Figure 2 shows the local structure of a two-dimensional free surface flow. To a first approximation, we can view the local base state free surface shape as the inner surface of a circular cylinder and impose a local cylindrical coordinate system as shown. Denoting base state quantities with overbars, the base state free surface position $\bar{h}$ is given by $r=\bar{h}$ $=R$. Thus $R$ is the local radius of curvature of the free surface and $\overline{\mathbf{n}}=-\mathbf{e}_{r}$ is the outward unit normal on the free surface. With $\overline{\boldsymbol{\sigma}}$ the total base state stress tensor and $g_{r}$ the local radial component of gravity, and neglecting inertia, the radial momentum balance here is

$$
0=\frac{1}{r} \frac{\partial}{\partial r}\left(r \bar{\sigma}_{r r}\right)+\frac{1}{r} \frac{\partial \bar{\sigma}_{\theta r}}{\partial \theta}-\frac{\bar{\sigma}_{\theta \theta}}{r}+\rho g_{r} .
$$

Anticipating the development below, we rearrange this, evaluate it at the free surface $r=R$, and apply the tangential stress boundary condition, $\bar{\sigma}_{\theta r}=0$, to yield the radial gradient of radial normal stress there:

$$
\frac{\partial \bar{\sigma}_{r r}}{\partial r}=\frac{\Delta \sigma}{R}-\rho g_{r},
$$

where $\Delta \sigma \equiv \bar{\sigma}_{\theta \theta}-\bar{\sigma}_{r r}$. The character of $\Delta \sigma$ in Newtonian and viscoelastic flows will be discussed below.

Now consider an infinitesimal perturbation to the free surface position, as shown in Fig. 3(a), so that $h=\bar{h}$ $-\hat{h}(z, \theta, t)$ and $\boldsymbol{\sigma}=\overline{\boldsymbol{\sigma}}+\hat{\boldsymbol{\sigma}}$. (Notice that we have defined $\hat{h}$ so that a positive value denotes an inward radial displacement, or local thickening, of the fluid layer.) We consider only 

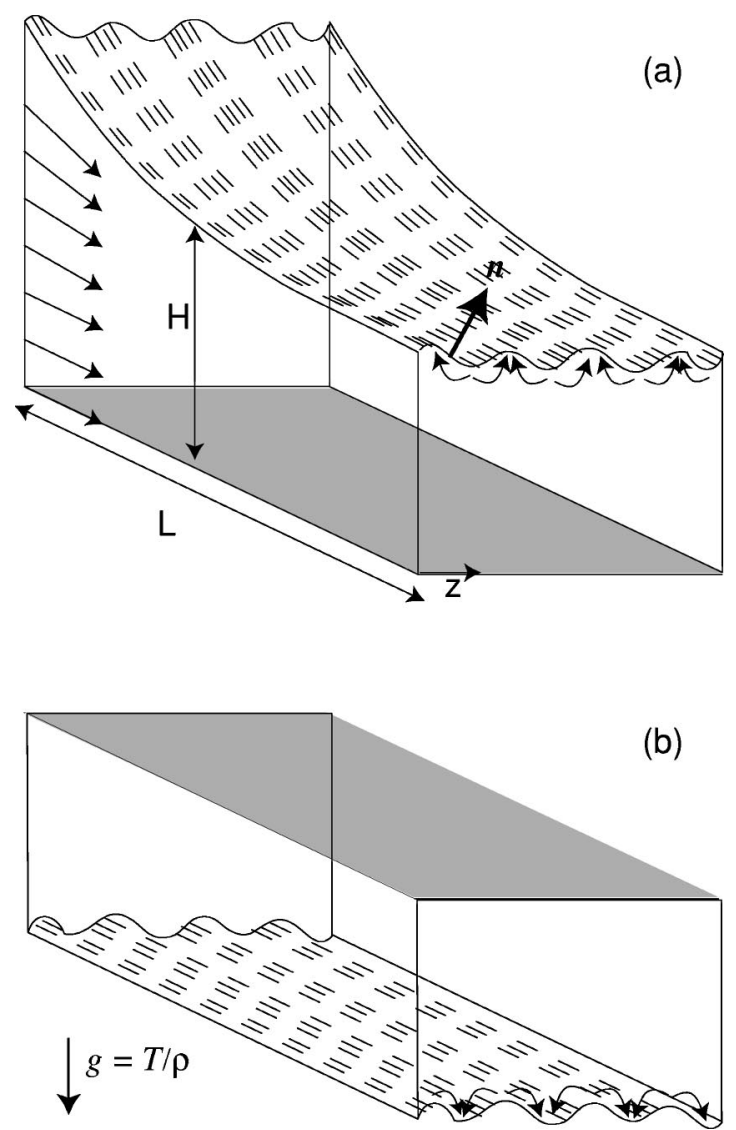

FIG. 3. (a) Schematic of a region of a planar coating flow subjected to a perturbation with significant spanwise variation but small streamwise variation. (b) Schematic of a fluid layer undergoing Rayleigh-Taylor instability.

perturbations with small streamwise variation in the interface position (i.e., || $1 / R \partial \hat{h} / \partial \theta\|\ll\| \partial \hat{h} / \partial z \|$ ), both because those are what are found to be unstable experimentally, and because of a priori considerations that will be discussed below. Expanding the stress field around the base state free surface position yields that

$$
\boldsymbol{\sigma}(\bar{h}-\hat{h})=\left.\overline{\boldsymbol{\sigma}}\right|_{\bar{h}}+\left.\hat{\boldsymbol{\sigma}}\right|_{\bar{h}}-\left.\hat{h} \frac{\partial \overline{\boldsymbol{\sigma}}}{\partial r}\right|_{\bar{h}}+O\left(\hat{h}^{2}\right) .
$$

The normal stress boundary condition is given by

$$
\mathbf{n} \cdot \boldsymbol{\sigma} \cdot \mathbf{n}=\gamma\left(\frac{1}{R_{1}}+\frac{1}{R_{2}}\right),
$$

where $R_{1}$ and $R_{2}$ are the principal radii of curvature of the interface, defined as positive if the center of curvature is in the phase into which $\mathbf{n}$ points. Applying these formulas along with Eq. (2) and neglecting the terms on the right-hand side (rhs) arising from the azimuthal curvature of the free surface yields

$$
\begin{aligned}
& \hat{\sigma}_{r r} \mid \bar{h} \approx T \hat{h}+\gamma \frac{\partial^{2} \hat{h}}{\partial z^{2}}, \\
& T \equiv \frac{\Delta \sigma}{R}-\rho g_{r} .
\end{aligned}
$$

Note that a positive value of $\left.\hat{\sigma}_{r r}\right|_{\bar{h}}$ indicates a radially inward traction exerted on the fluid at $r=\bar{h}$, that is, $\hat{\sigma}_{r r}\left|\bar{h}=\hat{\sigma}_{n n}\right| \bar{h}$. Let $T$ be positive. Then, if $\hat{h}$ is positive (i.e., the interface is locally deformed upward in Fig. 2), there is a net upward force at $r=\bar{h}$, which will pull fluid upward there, increasing $\hat{h}$ and making the upward force even larger. Likewise, for $\hat{h}<0$, the stress gradient tends to suck the interface into the fluid, making $\hat{h}$ more negative. This destabilizing tendency will be resisted by surface tension. A basic approximate criterion then, for free surface instability with a particular spanwise wave number $k$ is

$$
\frac{\Delta \sigma}{R}-\rho g_{r}>\gamma k^{2} .
$$

To be precise, this is really a local criterion, as $\Delta \sigma, R, g_{r}$ and $\hat{h}$ all vary in the $\theta$ direction, that is, along the free surface. Nevertheless, this criterion applies to any material—no constitutive information has yet been applied-and we view the form of the expression as indicative of the general mechanisms governing stability of free surface flows.

\section{NEWTONIAN AND VISCOELASTIC STABILITY CRITERIA}

Now let us consider some specific situations. If the fluid is Newtonian, then we can estimate $\Delta \sigma \approx c \eta U / H$, where $\eta$, $U$ and $H$ are the fluid viscosity and characteristic velocity and length scales for the flow, and $c=O(1)$. Defining capillary and Bond numbers $C a=\eta U / \gamma$ and $B o=\rho g_{r} H^{2} / \gamma$, and a dimensionless free surface curvature $K=H / R$, the above instability criterion becomes

$$
c \mathrm{CaK}-\mathrm{Bo}>\mathrm{k}^{2} \mathrm{H}^{2} \text {. }
$$

This is essentially a restatement of the Saffman-Taylor instability criterion, using the rhs of Eq. (2) in the perturbed boundary condition instead of the bulk pressure gradient, and for a fluid at rest it reduces to the Rayleigh-Taylor criterion. This formulation, however, brings out an interesting point: all else being fixed, at the instability threshold $C a \sim K^{-1}$.

Now we turn to how this criterion changes in a viscoelastic liquid. Consider a fluid with shear modulus $G$, relaxation time $\lambda$, total (zero-shear) viscosity $\eta$ and ratio of solvent to total viscosity $\beta$. The quantity $G / T$ defines an intrinsically elastic length scale $L_{e}$ whose physical significance will be illustrated in Sec. IV. Defining a Weissenberg number $W i=\lambda U / H$ and a dimensionless normal stress difference $\Sigma=\Delta \sigma / G$, the instability criterion, Eq. (7), becomes

$$
\frac{(1-\beta) C a}{W i} K \Sigma-B o>k^{2} H^{2} .
$$

[Note that $\Sigma \sim W i /(1-\beta)$ as $W i \rightarrow 0$, so Eq. (8) is recovered in the Newtonian limit.] The combination $(1-\beta) \mathrm{Ca} / \mathrm{Wi}$ $=G H / \gamma$, which we denote $\mathcal{N}$, is velocity-independent, and can be viewed as an elastocapillary number, and $K \Sigma$ is a dimensionless product of hoop stress and curvature identical to those that arise in criteria for viscoelastic bulk flow instabilities. ${ }^{31-34}$ In experiments with a given fluid, $\mathrm{Ca}$ and $W i$ are related: 


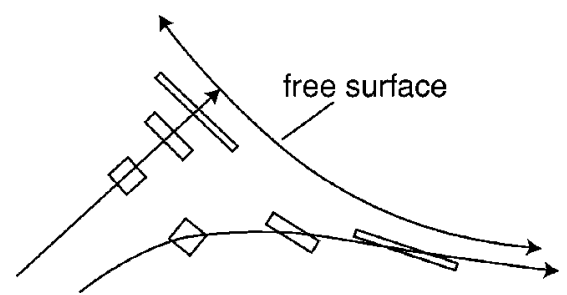

FIG. 4. Kinematics of fluid elements approaching a shear-free surface with a stagnation point, $\boldsymbol{\nabla}_{s} \cdot \mathbf{v}>0$.

$$
C a=\frac{\mathcal{N}}{1-\beta} W i .
$$

Noting that $\Sigma$ is a function of $W i$, the instability criterion, inequality (9) can be viewed as a locus of points on the $\mathrm{KCa}$ vs $W i$ plane. A set of experiments with increasing speed corresponds to motion on this plane upward along the curve defined by Eq. (10). Instability occurs at the intersection of the two curves; examination of $\Sigma$ and the underlying flow kinematics that determine it reveals why viscoelasticity destabilizes free surface flows.

The overall kinematic character of the flows in Fig. 1 is extensional- the fluid is drawn from a thicker layer to a thinner one, so fluid elements tend to stretch in the $\theta$ direction and contract in $r$. This extensional behavior provides a partial explanation for why interface disturbances do not have significant streamwise variation: to do so they would need to work against a base state flow field that acts not only to convect them through the flow domain, but also to pull them apart, stretching them exponentially in the streamwise direction. Furthermore, in order for the zero tangential stress condition to hold at the free surface, the flow there must be purely extensional. For Newtonian liquids, the stress is $O(\eta U / H)$ regardless of whether the flow is shear or extension, but for a highly elastic liquid, the extensional nature of the free surface flow can lead to stresses much larger than this if the extension rate $\dot{\epsilon}$ is sufficiently rapid and the residence time $t_{R}$ in the high extension region sufficiently long. For an Oldroyd-B fluid with $W i \gg 1, \Sigma \approx \exp \left(2 \dot{\epsilon}_{R}\right)$. Finally, some free surface flows have a stagnation point on the free surface-the forward roll coating flow of Fig. 1, for example, must have at least one stagnation point at which fluid elements stretch along the free surface (in other words, where $\boldsymbol{\nabla}_{s} \cdot \mathbf{v}>0$ ). On a planar shear-free surface, the stagnation line must be orthogonal to the free surface (otherwise it would not be shear-free); in this situation and with $\boldsymbol{\nabla}_{s} \cdot \mathbf{v}$ $>0$ Kumar and Graham ${ }^{35}$ showed how stress boundary layers will arise when the extension rate is sufficiently large. As a fluid element approaches the free surface, it stretches along the direction tangent to the free surface. The closer the fluid element is to the free surface, the longer it has been exposed to the extensional flow and the more highly stretched it will be, as illustrated in Fig. 4. Specifically,

$$
\text { strain } \sim(\text { distance from free surface })^{-1} \text {. }
$$

This simple kinematical observation is the source of free surface stress boundary layers. All of these general considerations are corroborated by recent computational and experi- mental studies of flows of elastic liquids, which indicate the presence of thin stress boundary layers at the free surface. ${ }^{27-30,36-38}$ In view of this discussion, an alternate form of the instability criterion can be given by replacing $\mathrm{Ca}$ in the Newtonian expression, Eq. (8) by $\mathrm{Ca} \eta_{e} / \eta$, where $\eta_{e}$ is a planar extensional viscosity appropriate for the characteristic strain and strain rate on the free surface. The importance of extensional viscosity in the destabilization of coating flows was recognized empirically by Glass and co-workers. ${ }^{4,6}$ The study of Varela López et al. ${ }^{7}$ compares critical capillary numbers for instability of Newtonian fluid with those for Xanthan solutions and high molecular weight $\left(12 \times 10^{6} \mathrm{~g} / \mathrm{mol}\right)$ polyacrylamide solutions. Xanthan is a stiff polymer, which will have small $\eta_{e} / \eta$, while high molecular weight polyacrylamide is very flexible, having large $\eta_{e} / \eta{ }^{39}$ The stability results are consistent with these trends; the critical $\mathrm{Ca}$ for the Xanthan solutions is lowered from the Newtonian by less than a factor of 2 , but that for the polyacrylamide solutions is nearly an order of magnitude lower.

Finally, the existence of stress boundary layers provides another a priori justification for neglecting streamwise variation of interface disturbances. The region of high streamwise tension near the free surface can be thought of as a membrane stretched in the mean flow direction. A stretched membrane strongly resists deformations with a wavevector in the direction of the stretching, leaving wavevectors transverse to the stretching unaffected. A clear illustration of how this mechanism operates in viscoelastic flows is given in the analysis by Hinch in the appendix of Ref. 40. It is shown there that through this mechanism, viscoelasticity mimics surface tension and suppresses short-wavelength KelvinHelmholtz instabilities of shear layers. The same mechanism will be present in viscoelastic free surface flows.

To summarize in mechanistic terms, an interface perturbation that locally thickens the fluid layer leads to a local accumulation of base state hoop stress, which pulls the material upward, driving further thickening of the layer. In elastic liquids, because of the large stresses that can arise in the extensional flow near the free surface, or equivalently the large extensional viscosity, the hoop stress can be quite large. As with viscoelastic bulk flow instabilities, ${ }^{34,41,42}$ for free surface flows the combination of tension and curvature is again the culprit. Indeed, this analysis shows that the same can be said even in the Newtonian case; however, tensions in Newtonian liquids are much smaller than those in elastic ones.

\section{GROWTH RATE AND WAVELENGTH SELECTION IN AN ANALYTICALLY SOLVABLE MODEL}

The above discussion illustrates the destabilization mechanism of flows with curved free surfaces. To further examine the consequences of this phenomenon, we focus on a situation that is amenable to analytical treatment. Our starting point is a fairly generic flow geometry: a region of a flow like that shown in Fig. 3(a), in the idealized case where the length $L$ of the region (which is comparable to the radius of curvature $R$ of the surface) is much larger than the typical thickness $H$. This is the basic structure of the "transition 
region" in Hele-Shaw and other coating flows, for example the downstream region in the slot coater on Fig. 1. (By focusing on the transition region, we do not mean to imply that the instability must originate there-it may originate near the stagnation point, but that region is not amenable to simplification to an analytically tractable form. We return to this issue below.) For the reasons described in the previous section, we only consider perturbations with a wavevector in the spanwise direction. For such longitudinal perturbations all convective terms will be negligible. Therefore a reasonable model of the stability of the flow in this region, in the limit $H / R \rightarrow 0$ with $(H / R)(\Delta \sigma / G)$ remaining finite, is simply the stability of a flat layer subject to the interfacial condition given by Eq. (5) - this is essentially a Rayleigh-Taylor problem, with gravity $g$ replaced by $T / \rho$ [Fig. 3(b)].

The solution to the Rayleigh-Taylor problem for an Oldroyd-B fluid is given by Aitken and Wilson; ${ }^{43}$ in this paragraph and the following we extract some key features of that analysis. All fluctuations that satisfy inequality (7) are unstable (as we argued above would be the case); thus as $T$ increases, long waves are the first to destabilize. The growth rate in this limit, $s_{\text {long }}$, satisfies

$$
s_{\text {long }}=\frac{\left(T-\gamma k^{2}\right) H^{3}}{3 \eta} k^{2} .
$$

Because $s_{\text {long }} \rightarrow 0$ as $k \rightarrow 0$, the time derivative term in the constitutive equation becomes negligible: thus the above result is the same as that for the purely viscous case. It is also of interest to consider the situation where $T \gg \gamma k^{2}$, where surface tension is negligible. For short waves $(k \gg T \lambda / \eta$ and $1 \ll k H \ll H \sqrt{T / \gamma}$ ), the viscous result is again recovered:

$$
s_{\text {short }}=\frac{T}{2 \eta k} \text {. }
$$

Although short waves grow due to the hoop stress at the surface, the growth rate vanishes as the wavenumber goes to infinity, even in the absence of surface tension.

Although at long and short wavelengths the Newtonian results are recovered, at intermediate wavelengths the growth rates in the viscoelastic and Newtonian cases can be quite different. Neglecting inertia and surface tension for simplicity, the dimensionless growth rate $\xi=s \eta / T H$ satisfies

$$
\frac{H}{L_{e}}(1-\beta) p \xi^{2}+\left(p-\frac{H}{L_{e}} \beta(1-\beta)\right) \xi-1=0,
$$

where

$$
p=\frac{2 k H\left((k H)^{2}+\cosh ^{2} k H\right)}{\sinh k H \cosh k H-k H}
$$

and $L_{e}=G / T$ is the elastic length scale introduced in Sec. III. The maximum growth rate (at $\gamma=0$ ) always occurs at $k H$ $\approx 2.11954$. (As surface tension increases, this is shifted to lower $k H$.) In the Newtonian case the scaled growth rate at this wavenumber is $\xi \approx 0.160698$. To illustrate what can happen in the viscoelastic case, Fig. 5 shows plots of $\xi$ vs wavenumber and (a) $L_{e} / H$ at $\beta=0.01$ and (b) $\beta$ at $L_{e} / H=0.1$, demonstrating that even for a fixed driving force $T$ and total viscosity $\eta$, the growth rate can be much larger for an elastic
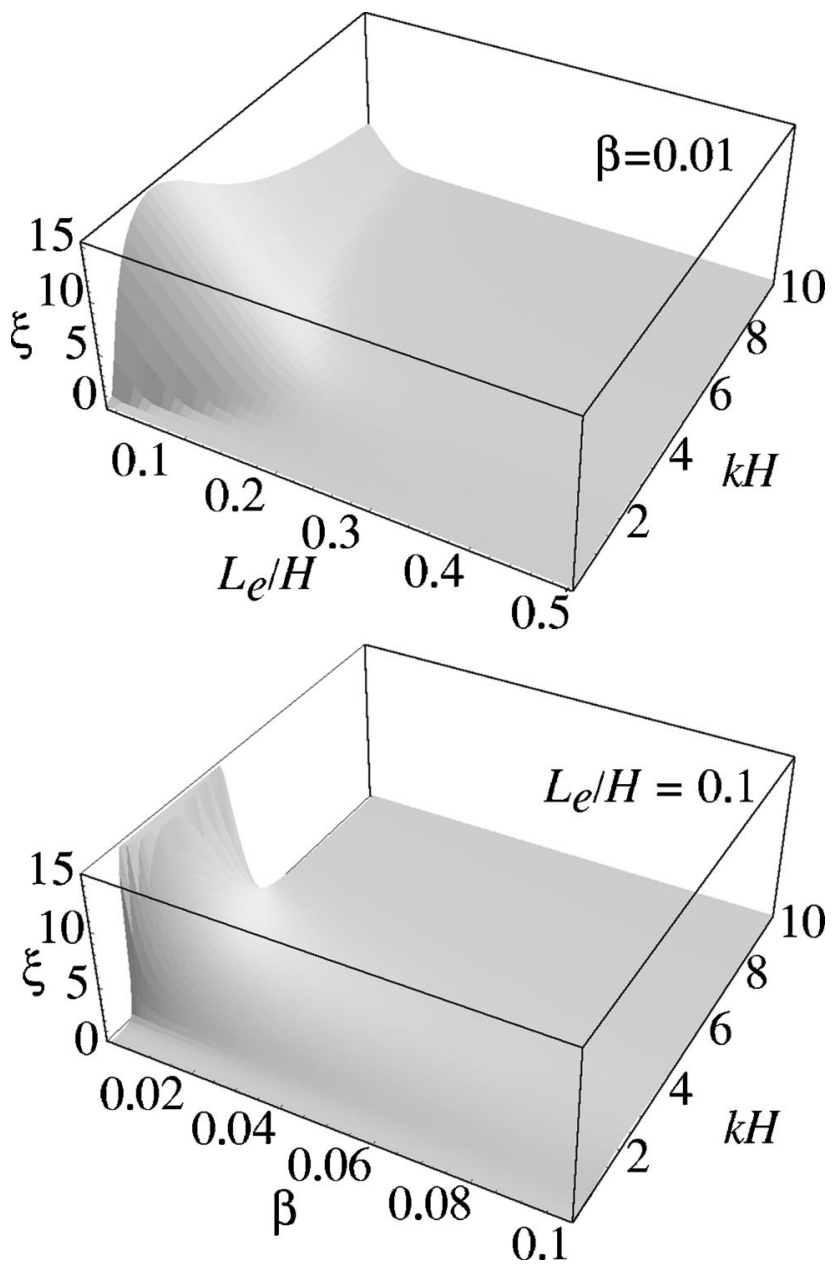

FIG. 5. Growth rates in inertialess viscoelastic Rayleigh-Taylor instability at (top) constant $\beta$ and (bottom) constant $L_{e} / H$.

liquid than for a Newtonian one. In particular, if $L_{e} / H$ $\lessgtr 0.16$, then there is a finite band of wavenumbers enclosing $k H=2.12$ where $L_{e} p / H<1$ and the growth rate satisfies

$$
\xi \approx\left(\frac{1}{p}-\frac{L_{e}}{H}\right) \beta^{-1} \text { as } \beta \rightarrow 0 .
$$

This result indicates that in this band of wavenumbers, the growth rate is controlled not by the total viscosity of the fluid, but only by the solvent contribution.

The singularity at $\beta=0$ is regularized by inertia (but not surface tension-it occurs at finite wavenumbers), as pointed out by Wilson. ${ }^{24,43}$ Experimental evidence of large growth rates was found by Joseph et al. ${ }^{44}$ in the breakup of viscoelastic drops subjected to shock waves. A mechanistic understanding of this singularity, and the instability phenomenon in general, can be gained by examining the energy balance. For the linearized equations of motion and boundary conditions, this is given by

$$
\dot{K}+\dot{U}=\dot{W}_{s},
$$

where 


$$
\begin{aligned}
& \dot{K} \equiv \frac{d}{d t} \int_{V} \frac{1}{2} \rho v^{2} d V, \\
& \dot{U} \equiv \int_{V} \boldsymbol{\sigma}: \nabla \mathbf{v} d V, \\
& \dot{W}_{s} \equiv \int_{S} \mathbf{v} \cdot(\mathbf{n} \cdot \boldsymbol{\sigma}) d S
\end{aligned}
$$

are, respectively, the rate of change of kinetic energy, rate of change of internal energy and rate of surface work done by the combination of interface deformation and base state stress gradient. The domain of integration is the region $\{0$ $<x<L, 0<y<H, 0<z \leqslant 2 \pi / k\}$. Because the exact solution is known, these terms can be evaluated exactly, but some insight can be gained by a heuristic analysis. The long wave limit is uninteresting - the surface work is balanced by viscous dissipation, so consider the situation $k H \gtrsim 1$. First, note that the solution for the surface displacement has the form $\hat{h}(z, t)=\widetilde{h} \exp (s t+i k z)$ and that the velocity in the fluid scales with $\partial \hat{h} / \partial t$, so $|\mathbf{v}| \sim s \hat{h}$ and || $\boldsymbol{\nabla} \mathbf{v}|| \sim k s \hat{h}$. Furthermore, $V \sim L / k^{2}$ and $S \sim L / k$. With these estimates,

$$
\dot{K} \sim \frac{\widetilde{h}^{2} s^{3} \rho L}{k^{2}} e^{2 s t}
$$

and [using Eq. (5)]

$$
\dot{W}_{s} \sim \frac{\left(T-\gamma k^{2}\right) s \widetilde{h}^{2} L}{k} e^{2 s t} .
$$

The rate of conversion to internal energy can be split into a contribution from the polymer stress, $\dot{E}$ and one from the viscous stress $\dot{D}$. For a rapidly growing perturbation, $\lambda s$ $\gg 1$, polymer relaxation can be neglected, and $\dot{E}$ becomes a stored strain energy contribution: the polymer contribution satisfies

$$
\dot{E} \sim s G \widetilde{h}^{2} L e^{2 s t}
$$

Finally, the viscous dissipation satisfies

$$
\dot{D} \sim \eta_{s} \widetilde{h}^{2} s^{2} L e^{2 s t} .
$$

If inertia and solvent viscosity are neglected, then the strain energy must balance the surface work, $\dot{E}=\dot{W}_{s}$. This balance can only hold if $k=\left(T-\gamma^{2} k^{2}\right) / G$ or, neglecting surface tension, $k L_{e}=1$. Thus we see the physical significance of $L_{e}$ : it is the disturbance wavelength at which surface work matches strain energy. For $\rho=0$ and $\eta_{s} \rightarrow 0$, dissipation and surface work balance when $k L_{e}<1$ giving

$$
s \sim \frac{T\left(1-k L_{e}\right)}{k \eta_{s}} .
$$

This is an approximate version of Eq. (14), showing the same singularity. We now see that this singularity arises at wavelengths where the strain energy stored in the deformation cannot balance the work done at the interface. For $\eta_{s}$ $=0$ and $\rho \rightarrow 0$, inertia balances surface work and we have

$$
s \sim \sqrt{\frac{T\left(1-k L_{e}\right) k}{\rho}} .
$$

For $G=0$ and $T=\rho g$, this last result reduces to that for the classical inviscid Rayleigh-Taylor instability, $s \sim \sqrt{g k} .45$

To summarize, instability occurs when $T>0$. As $T$ increases, increasingly short wavelength disturbances are excited, but the fastest growing wavelength is never shorter than $O(H)$. In particular, applying the above growth rate results locally on a free surface suggests that the instability will "originate" at the position where the product $T H$ is largest, where $T$ and $H$ measure the local normal stress gradient and fluid layer thickness, respectively. Without a detailed calculation, it cannot be said with certainty where this product will be maximized, but it seems likely that it will be in the stagnation point region rather than the transition region, because both the free surface curvature and effective layer thickness are larger near the stagnation point than downstream of it. In dimensionless terms, the approximate local selection criterion is

$$
k_{\max } H=O\left(\min \left(\frac{(1-\beta) C a}{W i} K \Sigma-B o, 1\right)\right) .
$$

This result is consistent, for example, with observations of Grillet et al. ${ }^{5}$ in displacement flows in an eccentric cylinder device, where the critical $\mathrm{Ca}$ for instability decreases as the fluid elasticity increases, and the observed wavenumber increases. Finally, if $G / T H \ll 1$, the growth rate is expected to scale inversely with the solvent viscosity rather than the total viscosity.

\section{APPLICATION TO FILAMENT STRETCHING INSTABILITY}

Now we apply this theory to develop a specific criterion for predicting the critical strain at which the filament stretching instability occurs. This flow is nominally axisymmetric rather than planar, and also inherently transient, but the overall picture presented here is not expected to be qualitatively sensitive to this complication. In particular we note that since the base state momentum balance and stress boundary condition hold instantaneously, Eq. (6) is valid for transient as well as steady flows.

To evaluate the instability criterion, inequality (7), three quantities need to be estimated: the stress difference, the wavelength of the instability and the radius of curvature. Spiegelberg and McKinley, ${ }^{9}$ in applying a similar theory to the experiments, estimated the stresses with the Oldroyd-B model. We will also do so here. For a filament stretching experiment with extension rate $\dot{\epsilon}$ and Weissenberg number $W i=\lambda \dot{\epsilon}>1 / 2$, we estimate that

$$
\Delta \sigma=\frac{2 G W i}{2 W i-1} \exp \left(\left(2-\frac{1}{W i}\right) \dot{\epsilon} t\right) .
$$

(Recall that the $\theta$-direction here is the direction tangent to the free surface in the flow direction.) Now, we need to choose a wavenumber at which to evaluate the criterion. Although the criterion is first satisfied for long waves, these are filtered out in the filament stretching device-wavelengths 
are quantized by the azimuthal periodicity, with the longest possible wavelength being $O\left(R_{f}\right)$ with $R_{f}$ the instantaneous filament radius. Furthermore the wavelength selection theory above predicts that once surface tension is overtaken, the fastest growing wavelength is on the order of the fluid layer thickness, which in the axisymmetric case would again be $R_{f}$. This prediction is consistent with the experimental observations; therefore we estimate that

$$
k=\frac{c_{1}}{R_{f}}=\frac{c_{1}}{R_{0}} \exp \left(\frac{\dot{\epsilon}}{2} t\right),
$$

where $R_{0}$ is radius of the endplates and $c_{1}$ is an $O(1)$ constant (or more precisely a weak function of experimental parameters). With this estimate for wavenumber the gravity term in Eq. (7) becomes negligible at large strains.

Finally, the appropriate radius of curvature in the $r-\theta$ plane ( $r-z$ in the usual coordinates for axisymmetric flow) must be evaluated. The curvature of the interface is highest where the filament joins the thin film near the endplate, where it can be estimated to be comparable to the radius of the filament, perhaps with a weak dependence on the initial aspect ratio of the fluid layer. It is not clear a priori, though, that the normal stress difference will be high in that region. Another possibility, then, is to posit the existence of a transition region on the filament, between the endplate region and the cylindrical bulk of the filament, in which the stress is large and the curvature small, with radius of curvature $R$ $=O\left(R_{0}\right)$. To include both possibilities we take

$$
R=c_{2} R_{0} \exp \left(-b \frac{\dot{\epsilon}}{2} t\right),
$$

where $c_{2}$ is again $O(1)$ and $b=1$ (highest stress in highcurvature region) or $b=0$ (highest stress in transition region). [For comparison, the end result of Spiegelberg and McKinley's theory-a combination of Saffman-Taylor and dimensional arguments-is recovered from the present theory by setting $k=c_{1} R_{0}^{-1}$ and $R=c_{2} R_{f}(b=1)$. With these choices, however, $c_{1}^{2} c_{2}$ must be $O\left(10^{4}\right)$ to approach the data.]

Using the above estimates, our instability criterion, expressed as critical Hencky strain $\epsilon_{c}=(\dot{\epsilon} t)_{c}$ becomes

$$
\epsilon_{c}=\left(1+\frac{b}{2}-\frac{1}{W i}\right)^{-1} \log \left(\frac{c_{1}^{2} c_{2}}{\mathcal{N}}\left(1-\frac{1}{2 W i}\right)\right),
$$

where $\mathcal{N}=G R_{0} / \gamma$. Note that the theory with $b=1$ (high stress in high curvature region) predicts that no instability occurs when $W i<2 / 3$, a threshold which agrees well with the data of Spiegelberg and McKinley-the lowest Wi at which instability is reported on their Fig. 11 is 0.70. Simulations of the filament stretching process by Rasmussen and Hassager ${ }^{27}$ show that at $W i=1$, the region of high stress does extend into the high curvature region, consistent with the choice $b=1$. In this case, the quantity $L_{e} / H$ reduces to $G / \Delta \sigma$; when $W i>1 / 2$ this becomes exponentially small with increasing $\epsilon$, indicating the possibility of large growth rates.

Figure 6 shows the predictions of Eq. (26) with $b=1$, evaluated for $c_{1}^{2} c_{2}=100$ and $\mathcal{N}=1.46,1.12$ and 2.23 , corre-

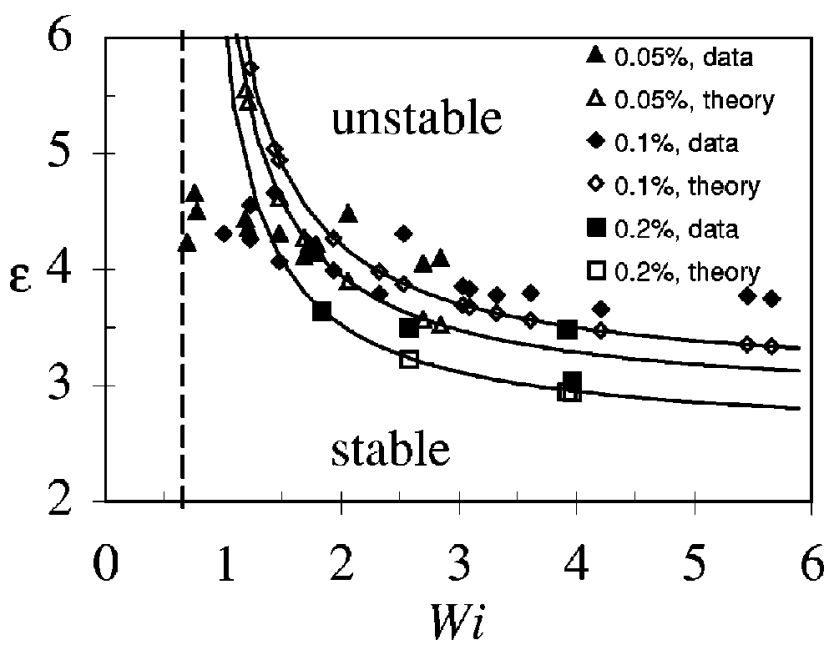

FIG. 6. Critical Hencky strain as a function of Weissenberg number. Lines and open symbols are evaluated from Eq. (26) with $b=1, c_{1}^{2} c_{2}=100$ and $\mathcal{N}=1.46,1.12$ and 2.23 , corresponding to the $0.05 \%, 0.1 \%$ and $0.2 \%$ PS solutions used by Spiegelberg and McKinley (Ref. 9). The solid symbols show their experimental results. The vertical dashed line is where $W i$ $=2 / 3$. The theory with $b=1$ predicts that the flow is always stable if $W i$ $<2 / 3$.

sponding to the $0.05 \%, 0.1 \%$ and $0.2 \%$ PS solutions used by Spiegelberg and McKinley, as well as the experimental data for those solutions. (Curiously, the value of $G$ extracted from their viscosity and first normal stress data is lower for the $0.1 \%$ solution than for the others). Although the theory captures the general trend of the data, it consistently overpredicts the critical strain at low $W i$, perhaps because the transient nature of the filament stretching process enhances disturbance growth, an effect the present quasistatic theory cannot capture.

Spiegelberg and McKinley also report the critical stress $(\Delta \sigma)_{c}$ for instability as a function of $W i$, showing that, at least above $W i \approx 1$, the critical stress is an increasing function of $W i$. The simple theory here gives

$$
(\Delta \sigma)_{c}=\frac{2 W i G}{2 W i-1}\left(\frac{c_{1}^{2} c_{2}}{\mathcal{N}}\left(1-\frac{1}{2 W i}\right)\right)^{(2 W i-1) /(W i(1+b / 2)-1)} .
$$

For $W i \gg 1$ and $c_{1}^{2} c_{2}=$ const, this result predicts a critical stress that is independent of $W i$. The increasing dependence found experimentally can be recovered if $c_{1}^{2} c_{2}$ is made an increasing function of $W i$. One possible source of such a dependence is a tendency for a filament of strain-hardening fluid to become more nearly cylindrical with increasing $W i$, as found in the simulations of Yao, Spiegelberg and McKinley, ${ }^{38}$ with the high stress region no longer extending "around the corner" into the high curvature region. An attempt to model this effect is beyond the scope of the present theory. In any case, the theory does predict that for $W i \gg 1$, $(\Delta \sigma)_{c}$ decreases as $G$ increases, consistent with the experiments, as well as capturing the critical Wi above which instability is observed. 


\section{CONCLUSION}

By generating stress gradients near concave free surfaces, the combination of free surface curvature and elastic normal stresses provides a driving mechanism for free surface instability in flowing elastic liquids. Because of the extensional nature of the flow on the free surface, these normal stresses can become quite large. For a particular idealized flow geometry, the resulting instability is analogous to the Rayleigh-Taylor instability of a viscoelastic fluid layer. In this case, a previous analysis ${ }^{43}$ can be used and extended to provide further insight into the instability. It is seen that once the stabilizing effect of surface tension is overcome, the fastest growing wavelength is of the order of the fluid layer thickness.

The theory developed here captures several features of experimental observations of coating flows in eccentric cylinder geometries, specifically the correlation between destabilization and extensional viscosity and the increase in wavenumber with viscoelasticity. Application of the theory to the instability of filament stretching flow yields a prediction of the Weissenberg number below which instability does not occur that agrees well with experimental observations. It is hoped that the simple arguments presented here will aid in organizing and understanding experimental observations and computational predictions of free surface instabilities in soft elastic materials.

\section{ACKNOWLEDGMENTS}

This research was supported in part by the National Science Foundation, Grants Nos. PHY-9907949 and EEC/BES/ CTS-0085560, and the donors of the Petroleum Research Fund. The author gratefully acknowledges the incisive comments of G. M. Homsy, as well as his hospitality and that of the Kavli Institute for Theoretical Physics at the University of California, Santa Barbara, where some of this work was performed. Gareth McKinley graciously provided the experimental data shown in Fig. 6.

${ }^{1}$ T. Bauman, T. Sullivan, and S. Middleman, "Ribbing instability in coating flows: Effect of polymer additives," Chem. Eng. Commun. 14, 35 (1982).

${ }^{2}$ D. J. Coyle, C. W. Macosko, and L. E. Scriven, "Reverse roll coating of non-Newtonian liquids," J. Rheol. 34, 615 (1990).

${ }^{3}$ P. Dontula, M. Pasquali, C. W. Macosko, and L. E. Scriven, "Viscoelastic effects in forward-roll coating flows," in Proc. XIIth Int. Congr. on Rheology (1996)

${ }^{4}$ R. H. Fernando and J. E. Glass, "Dynamic uniaxial extensional viscosity (DUEV) effects in roll applications II. Polymer blend studies," J. Rheol. 32, 199 (1988).

${ }^{5}$ A. M. Grillet, A. G. Lee, and E. S. G. Shaqfeh, "Observations of ribbing instabilities in elastic fluid flows with gravity stabilization," J. Fluid Mech. 399, 49 (1999).

${ }^{6}$ D. A. Soules, R. H. Fernando, and J. E. Glass, "Dynamic uniaxial extensional viscosity (DUEV) effects in roll applications I. Rib and web growth in commercial coatings," J. Rheol. 32, 181 (1988).

${ }^{7}$ F. Varela-López, L. Pauchard, M. Rosen, and M. Rabaud, "NonNewtonian effects on ribbing instability threshold," J. Non-Newtonian Fluid Mech. 103, 123 (2002).

${ }^{8}$ C.-Y. Ning, C.-C. Tsai, and T.-J. Liu, "The effect of polymer additives on extrusion slot coating," Chem. Eng. Sci. 51, 3289 (1996).

${ }^{9}$ S. H. Spiegelberg and G. H. McKinley, "Stress relaxation and elastic decohesion of viscoelastic polymer solutions in extensional flow," J. NonNewtonian Fluid Mech. 67, 49 (1998).

${ }^{10}$ T. Sridhar, V. Tirtaamadja, D. A. Nguyen, and R. K. Gupta, "Measurement of extensional viscosity of polymer solutions," J. Non-Newtonian Fluid Mech. 40, 271 (1991).

${ }^{11} \mathrm{~V}$. Tirtaamadja and T. Sridhar, "A filament stretching device for measurement of extensional viscosity," J. Rheol. 37, 1081 (1993).

${ }^{12}$ K. R. Shull, C. M. Flanigan, and A. J. Crosby, "Fingering instabilities of confined elastic layers in tension," Phys. Rev. Lett. 84, 3057 (2000).

${ }^{13}$ P. G. Saffman and G. I. Taylor, "The penetration of a fluid into a porous medium or Hele-Shaw cell containing a more viscous liquid," Proc. R. Soc. London, Ser. A 245, 312 (1958).

${ }^{14}$ G. M. Homsy, "Viscous fingering in porous media," Annu. Rev. Fluid Mech. 19, 271 (1987).

${ }^{15}$ C.-W. Park and G. M. Homsy, "Two-phase displacement in Hele-Shaw flows: Theory," J. Fluid Mech. 139, 291 (1984).

${ }^{16}$ L. Schwartz, "Stability of Hele-Shaw flows: The wetting layer effect," Phys. Fluids 29, 3086 (1986).

${ }^{17}$ J. R. A. Pearson, "The instability of uniform viscous flow under rollers and spreaders," J. Fluid Mech. 7, 481 (1960).

${ }^{18}$ D. J. Coyle, C. W. Macosko, and L. E. Scriven, "Stability of symmetric film-splitting between counter-rotating cylinders," J. Fluid Mech. 216, 437 (1990).

${ }^{19}$ I. D. Gates, Ph.D. thesis, University of Minnesota, 1999.

${ }^{20}$ L. C. Musson, Ph.D. thesis, University of Minnesota, 2001.

${ }^{21}$ L. Sartor, Ph.D. thesis, University of Minnesota, 1990.

${ }^{22}$ M. E. Gurfinkel-Castillo and A. T. Patera, "Three-dimensional ribbing instability in symmetric forward-roll film-coating processes," J. Fluid Mech. 335, 323 (1997).

${ }^{23}$ A. D. McEwan and G. I. Taylor, "The peeling of a flexible strip attached by a viscous adhesive," J. Fluid Mech. 26, 1 (1966).

${ }^{24}$ S. D. R. Wilson, "The Taylor-Saffman problem for a non-Newtonian liquid," J. Fluid Mech. 220, 413 (1990).

${ }^{25}$ J. S. Ro and G. M. Homsy, "Viscoelastic free surface flows: Thin film dynamics of Hele-Shaw and dip coating flows," J. Non-Newtonian Fluid Mech. 57, 203 (1995).

${ }^{26}$ A. M. Grillet, A. C. B. Bogaerds, G. W. M. Peters, and F. P. T. Baaijens, "Numerical analysis of flow mark surface defects in injection molding flow," J. Rheol. 93, 651 (2002).

${ }^{27}$ H. K. Rasmussen and O. Hassager, "Three-dimensional simulations of viscoelastic instability in polymeric filaments," J. Non-Newtonian Fluid Mech. 82, 189 (1999).

${ }^{28} \mathrm{H}$. K. Rasmussen and O. Hassager, "The role of surface tension on the elastic decohesion of polymeric filaments," J. Rheol. 45, 527 (2001).

${ }^{29}$ A. G. Lee, E. Shaqfeh, and B. Khomami, "A study of viscoelastic free surface flows by the finite element method: Hele-Shaw and slot coating flows," J. Non-Newtonian Fluid Mech. 108, 327 (2002).

${ }^{30}$ M. Pasquali and L. E. Scriven, "Free surface flows of polymer solutions with models based on the conformation tensor," J. Non-Newtonian Fluid Mech. 108, 363 (2002).

${ }^{31}$ M. D. Graham, "Effect of axial flow on viscoelastic Taylor-Couette instability," J. Fluid Mech. 360, 341 (1998).

${ }^{32}$ Y. L. Joo and E. S. G. Shaqfeh, "Viscoelastic Poiseuille flow through a curved channel: A new elastic instability," Phys. Fluids A 3, 1691 (1991).

${ }^{33}$ R. G. Larson, E. S. G. Shaqfeh, and S. J. Muller, "A purely elastic instability in Taylor-Couette flow,” J. Fluid Mech. 218, 573 (1990).

${ }^{34}$ P. Pakdel and G. H. McKinley, "Elastic instability and curved streamlines," Phys. Rev. Lett. 77, 2459 (1996).

${ }^{35}$ K. A. Kumar and M. D. Graham, "Buckling instabilities in models of viscoelastic free surface flows," J. Non-Newtonian Fluid Mech. 89, 337 (2000).

${ }^{36}$ T. Podgorski and A. Belmonte, "Surface folds during the penetration of a viscoelastic fluid by a sphere," J. Fluid Mech. 460, 337 (2002).

${ }^{37}$ M. Yao and G. H. McKinley, "Numerical simulation of extensional deformations of viscoelastic liquid bridges in filament stretching devices," J. Non-Newtonian Fluid Mech. 74, 47 (1998).

${ }^{38}$ M. Yao, S. H. Spiegelberg, and G. H. McKinley, "Dynamics of weakly strain-hardening fluids in filament stretching devices," J. Non-Newtonian Fluid Mech. 89, 1 (2000).

${ }^{39}$ R. B. Bird, C. F. Curtiss, R. C. Armstrong, and O. Hassager, Dynamics of Polymeric Liquids, 2nd ed. (Wiley, New York, 1987), Vol. 2.

${ }^{40}$ J. Azaiez and G. M. Homsy, "Linear stability of free shear flow of viscoelastic liquids," J. Fluid Mech. 268, 37 (1994).

${ }^{41}$ R. G. Larson, "Instabilities in viscoelastic flows," Rheol. Acta 31, 213 (1992). 
${ }^{42}$ E. S. G. Shaqfeh, "Purely elastic instabilities in viscometric flows," Annu. Rev. Fluid Mech. 28, 129 (1996).

${ }^{43}$ L. S. Aitken and S. D. R. Wilson, "Rayleigh-Taylor instability in elastic liquids," J. Non-Newtonian Fluid Mech. 49, 13 (1993).
${ }^{44}$ D. D. Joseph, G. S. Beavers, and T. Funada, "Rayleigh-Taylor instability of viscoelastic drops at high Weber numbers," J. Fluid Mech. 453, 109 (2002).

${ }^{45}$ S. Chandrasekhar, Hydrodynamic and Hydromagnetic Stability (Dover, New York, 1981). 